\title{
Análise do preenchimento de prontuários odontológicos: questões éticas e legais
}

\author{
Analysis of the completion of dental records: ethical and legal issues \\ Análisis de la cumplimiento de expedientes dentales: cuestiones éticas y legales
}

\author{
Naiana Pereira Firmino De Oliveira \\ ORCID: https://orcid.org/0000-0001-7805-3995 \\ Centro Universitário de João Pessoa, Brasil \\ E-mail: naianacaiana@ hotmail.com \\ Yasmin Caldas de Macêdo Abrantes Rodrigues de Oliveira \\ ORCID: https://orcid.org/0000-0003-4122-2024 \\ Universidade Federal da Paraíba, Brasil \\ E-mail: yasmincmar@gmail.com \\ Bianca Oliveira Tôrres \\ ORCID: https://orcid.org/0000-0002-7256-2577 \\ Centro Universitário de João Pessoa, Brasil \\ E-mail: biancaotorres@yahoo.com.br \\ Solange Soares da Silva Felix \\ ORCID: https://orcid.org/0000-0001-9061-8717 \\ Universidade Federal da Paraíba, Brasil \\ E-mail:lannefelix@yahoo.com.br \\ Mara Ilka Holanda De Medeiros Batista \\ ORCID: https://orcid.org/0000-0002-7314-0595 \\ Faculdade Nova Esperança, Brasil \\ E-mail: marailka@hotmail.com
}

\begin{abstract}
Resumo
Introdução: Prontuários odontológicos são documentos diariamente produzidos por cirurgiões-dentistas e possuem grande valor administrativo e legal, devendo ser preenchidos com máxima cautela. Para isso foram analisados o preenchimento dos prontuários odontológicos produzidos no atendimento de pacientes no curso de Odontologia de uma universidade particular, tendo em vista as questões éticas e legais que norteiam o preenchimento da referida documentação. Metodologia: Foram analisados 350 prontuários através do uso de um formulário contendo questões referentes aos dados obrigatórios para o correto preenchimento dos principais aspectos do prontuário. Os dados coletados foram analisados a partir da estatística descritiva utilizando o programa estatístico Statistical Package for the Social Sciences - SPSS, versão 20.0. Resultados: A maioria dos prontuários analisados apresentavam todos os dados de identificação do paciente. Apenas 34,9\% tinham a anamnese preenchida de forma completa, $21,4 \%$ dos casos o plano de tratamento estava escrito de forma completa, haviam radiografias anexadas ao prontuário em $77,1 \%$ da amostra, e 91,1\% apresentavam o registro de atividades completo. Quanto a assinatura do aluno, estava presente no em $95,7 \%$ dos prontuários, enquanto $93,7 \%$ continham a assinatura do professor. $4 \%$ dos prontuários apresentaram cópia de receituário/atestado anexado e nenhum dos prontuários tinham as orientações cirúrgicas anexadas. Em $22 \%$ estava presente em anexado o Termo de Consentimento Livre e Esclarecido e em apenas 18,9\% deles constava a assinatura do paciente. Conclusão: Ainda há falta de atenção por parte dos alunos e professores quanto aos cuidados de preenchimento do prontuário, já que alguns dos critérios para um correto preenchimento não foram atendidos.
\end{abstract}

Palavras-chave: Prontuário odontológico; Ética odontológica; Legislação odontológica.

\begin{abstract}
Introduction: Dental records are documents produced daily by dentists and have great administrative and legal value, and should be filled out with utmost care. For this purpose, the filling of dental records produced in the care of patients in the Dentistry course of a private university was analyzed, in view of the ethical and legal issues that guide the filling out of said documentation. Methodology: 350 medical records were analyzed using a form containing questions referring to mandatory data for the correct filling of the main aspects of the medical record. The collected data were analyzed using descriptive statistics using the Statistical Package for Social Sciences - SPSS, version 20.0. Results: Most of the medical records analyzed had all patient identification data. Only 34.9\% had the anamnesis completely filled out, $21.4 \%$ of the cases had the treatment plan written in full, there were radiographs attached to the medical record in $77.1 \%$ of the sample, and $91.1 \%$ had the record of full activities. As for the student's signature, it was present in $95.7 \%$ of the records, while $93.7 \%$ contained the teacher's signature. $4 \%$ of the medical records had a copy of the prescription/certificate attached and none of the medical records had the surgical guidelines attached. In $22 \%$, the Free and Informed Consent Form was attached, and only $18.9 \%$ of them contained the patient's signature.
\end{abstract}


Conclusion: There is still a lack of attention on the part of students and teachers regarding the care of filling out the medical record, as some of the criteria for a correct filling out were not met.

Keywords: Dental record; Dental ethics; Dental legislation.

\begin{abstract}
Resumen
Introducción: Los registros dentales son documentos elaborados diariamente por los dentistas y tienen un gran valor administrativo y legal, y deben ser llenados con sumo cuidado. Para ello, se analizó el llenado de expedientes odontológicos producidos en la atención de pacientes en el curso de Odontología de una universidad privada, atendiendo a las cuestiones éticas y legales que orientan el llenado de dicha documentación. Metodología: Se analizaron 350 historias clínicas mediante un formulario que contiene preguntas referentes a datos obligatorios para el correcto llenado de los principales aspectos de la historia clínica. Los datos recolectados fueron analizados usando estadística descriptiva usando el Paquete Estadístico para Ciencias Sociales - SPSS, versión 20.0. Resultados: La mayoría de las historias clínicas analizadas tenían todos los datos de identificación del paciente. Solo el 34,9\% tenía la anamnesis completa, el $21,4 \%$ de los casos tenía el plan de tratamiento redactado en su totalidad, había radiografías adjuntas a la historia clínica en el $77,1 \%$ de la muestra y el $91,1 \%$ tenía el registro de actividades completas. En cuanto a la firma del alumno, estaba presente en el 95,7\% de los expedientes, mientras que en el 93,7\% figuraba la firma del profesor. El 4\% de las historias clínicas tenía adjunta una copia de la prescripción / certificado y ninguna de las historias clínicas tenía adjuntadas las pautas quirúrgicas. En el $22 \%$ se adjuntó el Formulario de Consentimiento Libre e Informado, y solo el 18,9\% de ellos contenía la firma del paciente. Conclusión: Aún existe una falta de atención por parte de estudiantes y docentes con respecto al cuidado del llenado de la historia clínica, ya que no se cumplieron algunos de los criterios para un correcto llenado.
\end{abstract}

Palabras clave: Registro dental; Ética dental; Legislación dental.

\title{
1. Introdução
}

Os documentos odontológicos são um conjunto de declarações produzidas pelo cirurgião-dentista no exercício de sua função, uma verdadeira coletânea, firmadas pelo profissional e o paciente, no âmbito clínico, que servem como prova legal, podendo ser utilizados com finalidade jurídica e administrativa. Essa documentação é produzida, preenchida, atualizada e arquivada diariamente nos consultórios e clínicas escolas de instituições públicas ou privadas. A mesma é formada por anamnese, termo de consentimento livre e esclarecido, evolução clínica do tratamento, fotografias e radiografias do paciente, cópias de receituários e atestados e outros materiais produzidos ao longo do tratamento de cada paciente (Lima amorim et al., 2016).

Os prontuários odontológicos, na maioria das vezes, apresentam finalidade clínica, meramente administrativa, e oferecem condições ao profissional de consultar, a qualquer momento, os procedimentos realizados bem como o estágio em que se encontra o tratamento. Contudo, esses documentos podem subsidiar casos de identificação humana, pelo uso das informações registradas nas fichas em questão. O correto preenchimento do prontuário odontológico e a manutenção da sua integridade, possibilitam o seu uso em situações de necessidade pericial, sendo imprescindível para o processo de comparação nas perícias odontolegais. Por outro lado, a confecção deficiente, ou equivocada, pode levar a erros, e até mesmo impossibilitar a correta identificação da vítima (Mota et al., 2018). Hupp (2009) afirma que o registro deficiente de informações, rasuras e a falta de assinatura do TCLE, são os erros mais frequentemente encontrados nos prontuários, quando requeridos mediante demanda judicial.

O prontuário consta de toda documentação padronizada e destinada ao registro de informações pertinentes ao usuário de saúde, do início ao final do tratamento, composto por ficha clínica, plano de tratamento, radiografias, fotografias, modelos de gesso e outros exames complementares, evolução do tratamento, contrato de prestação de serviços odontológicos, atestado odontológico, termo de consentimento livre e esclarecido - TCLE e outros. O termo "Ficha Clínica" foi durante muito tempo utilizado para designar o nome de toda a documentação odontológica, mas considerando os aspectos éticos, legais e clínicos, o termo torna-se impróprio, devido ao grande número de informações que se deve ter sobre o paciente. Sendo assim, o conceito mais apropriado para a documentação odontológica passa a ser Prontuário Odontológico, pois este, junto com as informações referentes ao atendimento do paciente (ficha clínica), inclui toda a documentação que envolve o processo de atendimento. 
Nessa perspectiva, a ficha clínica passa a ser apenas um dos componentes do prontuário odontológico (Saraiva, 2012).

E de acordo com Vanrell (2016), embora na posse e sob guarda do cirurgião-dentista, o real proprietário dessa documentação original é o paciente, podendo o mesmo requer para utilizar como prova em processos judiciais, pois nele constam informações importantes de diagnóstico e tratamento utilizados, bem como o prognóstico e eventuais intercorrências, podendo o cirurgião-dentista permanecer com uma cópia em seu arquivo físico ou digital.

Segundo Kato et al. (2018), tratamento odontológico, seja em consultório, ou em clínicas de instituições de ensino, configura uma prestação de serviços e como tal é regida pelo Código Civil Brasileiro e pelo Código de Defesa do Consumidor. Assim, o paciente tem direitos assegurados e que devem ser preservados. Quando o tratamento é feito pelo aluno, o professor que o supervisiona é o responsável pelo tratamento executado. Por tudo isso, o prontuário odontológico deve ser bem estruturado, para que somente assim, torne-se o melhor instrumento que o profissional ou professor responsável pelo aluno têm ao seu alcance para produzir as provas necessárias à sua defesa, desde que esse documento contenha os dados necessários e suficientes para prestar todos os esclarecimentos que se fizerem necessários. Civilmente, o docente responsável pelo acompanhamento da atividade odontológica, responderá em juízo caso o paciente sinta-se prejudicado e acione o judiciário por procedimento realizado em desacordo com o proposto ou por procedimento que resulte danos. Caso o acionamento seja por dano moral decorrente de ofensa, ou qualquer outra prática não odontológica, o aluno também poderá responder, sendo civilmente capaz (Ferreira cruz et al.,2017).

Com base no exposto o presente estudo objetivou avaliar o preenchimento dos prontuários odontológicos de pacientes atendidos na clínica universitária em relação às questões éticas e legais preconizadas para o preenchimento da documentação odontológica, visando contribuir com a comunidade científica, profissionais, acadêmicos e à sociedade como um todo, acerca da importância da confecção do prontuário odontológico. Diante da relevância dessa documentação, o estudo se justifica, pois, a identificação de falhas no preenchimento das fichas clínicas odontológicas de pacientes é de suma importância para o aprimoramento e incentivo na elaboração e atualização do prontuário odontológico, visando aprimorar a eficiência técnica da clínica e prevenir processos judiciais.

\section{Metodologia}

Por se tratar de uma pesquisa envolvendo seres humanos foram atendidos todos os requisitos propostos pelo Conselho Nacional de Saúde/Ministério da Saúde, por meio da Resolução n 466/12. Este estudo é caracterizado como pesquisa aplicada, transversal, descritiva, documental, com abordagem quantitativa, realizada no arquivo de prontuários nas dependências da Clínica Escola do curso de Odontologia do Centro Universitário de João Pessoa - UNIPÊ. O universo foi composto por todos os prontuários odontológicos $(n=1000)$ produzidos na clínica escola de odontologia referentes aos semestres 2017.1 e 2017.2 do Centro Universitário de João Pessoa - UNIPÊ. E a amostra foi composta por 350 prontuários após aplicação dos critérios de inclusão e exclusão. Sendo assim, foram incluídos os prontuários elaborados nas clínicas nos semestres 2017.1 e 2017.2 que estavam devidamente cadastrados e arquivados em arquivos próprios na Clínica Escola de Odontologia do UNIPÊ. E foram excluídos todos os Prontuários de pacientes atendidos exclusivamente em casos de urgências e atendimento de triagens, pois possuem prontuário específico.

Para a realização da pesquisa foi utilizado como instrumento de coleta de dados um formulário próprio, sendo composto pelos itens a serem avaliados na documentação odontológica como: presença da identificação do paciente; preenchimento da anamnese; presença do plano de tratamento desenvolvido ou a ser desenvolvido; exames complementares quando necessário; radiografias para auxílio no diagnóstico quando necessário; preenchimento do registro de atividades desenvolvida com assinaturas do aluno e professor; cópia de atestados e receituários quando necessários; orientações pósoperatórias quando necessário; presença do TCLE e a sua assinatura pelo paciente ou responsável legal. Se aplica ainda a 
presença de rasuras.

Após a coleta e tabulação dos dados, os mesmos foram analisados estatisticamente de maneira descritiva utilizando o programa Statistical Package for the Social Sciences - SPSS, versão 20.0. Os resultados obtidos foram dispostos na forma de gráficos e tabelas de frequência absolutas e percentuais.

\section{Resultados e Discussão}

De acordo com as informações coletadas nos 350 prontuários odontológicos analisados, verificou-se a presença do nome do paciente estava em 99,4\% dos prontuários, do sexo (94\%), da idade (95,7\%), do telefone $(91,1 \%)$ e do endereço (92\%). O dado que menos apareceu registrado foi o número de telefone. Infere-se, portanto, que a maioria dos prontuários verificados continham as informações supracitadas de forma satisfatória. Em estudo realizado por Mota (2018), foi verificado que a correta confecção do prontuário odontológico e a manutenção da sua integridade, possibilitam o seu uso em situações de necessidade de identificação humana, sendo imprescindível para o sucesso da perícia odontolegal. Portanto, a confecção deficiente, ou equivocada, pode levar a erros, e até mesmo impossibilitar a correta identificação da vítima.

Um estudo realizado por Paranhos et al. (2009), ressalta a importância do prontuário odontológico nas perícias de identificação humana, quando realizou uma investigação com dados coletados dos registros do Laboratório de Antropologia Forense e Odontologia Legal do Instituto Médico Legal de Santo André- São Paulo, referentes aos anos de 2000 e 2007. Dos laudos periciais analisados $(n=32)$, apenas $37,50 \%$ foram identificados, dos quais $66,67 \%$ o foram por meio de prontuário odontológico, mostrando que possui relevância clínica e pericial por subsidiar com mais conteúdo informações relativas aos pacientes tratados, podendo, dessa forma, afirmar a identidade do cadáver.

As informações retiradas dos prontuários no presente estudo, apresentaram anamnese preenchida de forma completa em $34,9 \%$, incompleta em $62,9 \%$, e rasuras estavam presentes em 1,4\% deles. A anamnese deve ser realizada da forma detalhada e sem rasuras, facilitando o diagnóstico. As perguntas podem ser abertas, fechadas e direcionadas (Guimarães; Cariello; Almeida, 1994). Uma pesquisa desenvolvida por Carneiro-Neto et al. (2008) contou com 91 questionários respondidos por alunos do mestrado em ortodontia do Centro de Estudos Odontológicos São Leopoldo Mandic. Os autores constataram que $80,22 \%$ dos profissionais realizam anamnese, sendo que destes $23,08 \%$ não fazem registro por escrito dos dados coletados; 43,96\% não utilizam odontograma; 46,16\% e não fazem o registro das condições pré-clínicas. Quanto ao preenchimento do plano de tratamento, verificou-se que o mesmo estava ausente em $61,4 \%$ dos prontuários, enquanto que 21,4\% estavam presentes. Os incompletos somaram 17,1\%. Para Costa et al. (2009), é dever do profissional e direito do paciente ter informações claras sobre o tratamento, os riscos, as possibilidades e procedimentos executados. Portanto, um dos principais deveres do profissional no âmbito do atendimento ao paciente é o da informação, sendo a principal conduta cobrada frente à contestação de possíveis irregularidades. O direito à informação hoje é classificado como direito básico do consumidor. Nesse contexto, o plano de tratamento deverá conter, de forma detalhada, todos os procedimentos propostos e realizados, bem como o tipo de materiais utilizados. As opções de tratamento, as escolhas do paciente e os casos de discordância entre paciente e profissional devem ser registrados.

Foram observados que $77,1 \%$ dos prontuários avaliados continham algum tipo de raio-x anexado para auxilio no diagnóstico, já 16,9\% mostram não haver necessidade de solicitação desse tipo de exame. Não haviam radiografias anexadas em 6\% dos prontuários. Entre os exames complementares mais realizados nas Clinicas escolas e consultórios odontológicos são as radiografias. É importante ressaltar a relevância e obrigatoriedade de elaborar e conservar em arquivo próprio a documentação radiográfica, pois na falta de outras informações clínicas odontológicas por falhas da documentação clínica do cirurgião-dentista da vítima ou por mutilações ocorridas no cadáver, uma simples radiografia periapical pode fornecer dados clínicos suficientes para o processo de identificação (Souza et al., 2021). 
No tocante ao preenchimento completo do registro de atividades, 91,1\% dos prontuários apresentaram registro completo, registro ausente somou 6\%, já o registro incompleto totalizou 2,9\%. Para os autores Ditterich et al. (2008), todos os procedimentos realizados durante o tratamento odontológico, seja em consultório, seja em clínica de instituições de ensino, devem ser anotados na ficha clínica, pois os mesmos configuram prestação de serviços e como tal é regida pelo Código Civil Brasileiro e pelo Código de Defesa do Consumidor. Assim, o paciente tem direitos assegurados e que devem ser preservados. Quando o tratamento é feito pelo aluno, o professor que o supervisiona é o responsável pelo tratamento executado.

Por tudo isso, o autor supracitado ainda afirma que o prontuário odontológico deve ser bem estruturado, para que somente assim, torne-se o melhor instrumento que o profissional ou professor responsável pelo aluno têm ao seu alcance para produzir as provas necessárias à sua defesa, caso seja requerido judicialmente, desde que esse documento contenha os dados necessários e suficientes para prestar todos os esclarecimentos que se fizerem necessários (Ditterich et al., 2008). Na avaliação das assinaturas de alunos e professores constantes no registro de atividades, os resultados variaram conforme expressa a tabela 6 na qual 95,7\% dos prontuários analisados apresentavam a assinatura do aluno e 93,7\% apresentavam a assinatura do professor. Os dados da pesquisa expressam que alunos e professores estão cumprindo de forma satisfatória a obrigatoriedade das respectivas assinaturas no registro de atividades. Tendo em vista, que o profissional ao não assumir a responsabilidade pelos procedimentos clínicos realizados pelos alunos, em âmbito da instituição, comete infração ética segundo o Código de Ética Odontológica, podendo ser penalizado segundo as penas previstas na Lei 4.324/64 (BRASIL, 1964). Civilmente, o professor responsável pelo acompanhamento da atividade odontológica, responderá em juízo caso o paciente sinta-se prejudicado e acione o judiciário por procedimento realizado em desacordo com o proposto ou por procedimento que resulte danos. Caso o acionamento seja por dano moral decorrente de ofensa, ou qualquer outra prática não odontológica, o aluno também poderá responder, sendo civilmente capaz (Nogueira et al., 2014).

Quanto à questão da presença de cópias de atestados e receituários anexadas à documentação odontológica, em 59,7\% não houve necessidade das mesmas, já 36,3\% estavam ausentes e apenas 4\% estavam presentes. Para Ditterich et al. (2008) os atestados e prescrições de medicamentos devem ser elaborados da maneira correta e apenas em caso de real necessidade. As cópias devem ser arquivadas junto ao prontuário, mas é imprescindível que sejam carbonadas. Além disso, não se pode esquecer a necessidade da aposição do carimbo que deve, obrigatoriamente, pertencer àquele que está assinando a receita ou atestado. Em relação à presença das orientações cirúrgicas pós-operatórias dadas ao paciente pelo aluno, em 59,4\% dos prontuários avaliados não houve necessidade delas, em 40,6\% essas orientações estavam ausentes e em nenhum dos prontuários havia a presença dessas orientações.

O Termo de Consentimento Livre e Esclarecido estava ausente em $78 \%$ dos prontuários analisados, e presente em $22 \%$, no entanto, destes presentes apenas 18,9\% estavam assinados. O estudo realizado por Costa et al. (2009), diverge do presente estudo quanto à questão da assinatura do TCLE. O mesmo avaliou falhas no preenchimento das fichas clínicas odontológicas dos prontuários de pacientes atendidos na Universidade Estadual de Montes Claros, em 2005, sob aspectos éticos e legais. Foram analisados 881 prontuários, dos quais 94,9\% estavam assinados. Hupp (2009) afirma que o registro deficiente de informações, rasuras e a falta de assinatura do TCLE, são os erros mais frequentemente encontrados nos prontuários, quando requeridos mediante demanda judicial.

\section{Conclusão}

Conclui-se que, dos prontuários analisados foi possível constatar que na maioria deles os dados de identificação do paciente estavam preenchidos a contento. Identificou-se ainda que em sua maioria não constava em anexo o TCLE nem a assinatura do paciente ou responsável legal. Ao examinar as anotações dos tratamentos realizados, identificou-se que a maioria dos prontuários apresentavam esse quesito preenchido de forma completa. Dos prontuários envolvidos na pesquisa, a maioria 
apresentava a assinatura do aluno no registro de atividades (tratamento realizado); ainda foi observado que no registro de atividades (tratamento realizado), a assinatura do professor estava presente na maioria dos casos.

Diante disso, é necessário que a equipe de professores, alunos e funcionários, tenham mais atenção ao preenchimento dos prontuários, para que tudo esteja bem completo. Isso é muito importante para o bom funcionamento das clínicas, assim como para que a instituição fique resguardada quanto a documentação dos pacientes atendidos, já que se sabe que o prontuário odontológico pertence ao paciente, mas que fica sob guarda dos profissionais e instituições.

Reforçar aos colegas da área a importância do prontuário é sempre relevante, e mais trabalhos podem ser conduzidos nesse sentido a fim de elevarmos a qualidade do trabalho odontológico.

\section{Referências}

Almeida, S. M., Dwlwing, F., Azevedo, J. A. P., Nogueira, R. K. T., Falcão, F. P. \& Carvalho, S. P. M. (2015). Effectiveness of dental records in human identification. RGO-Revista Gaúcha de Odontologia, 63, 502-6.

Brasil. (1966). Lei no 5.081, de 24 de agosto de 1966. Regula o exercício da Odontologia no Brasil. http://www.planalto.gov.br/ccivil_03/Leis/L5.081.

Brasil. (2019). Res. CNS n ${ }^{\circ}$ 466, de 12 de dezembro de 2012. Aprova diretrizes e normas regulamentadoras de pesquisas envolvendo seres humanos. http://bvsms.saude.gov.br/bvs/saudelegis/cns/2013/res0466_12_12_2012.html

Carneiro Neto, H., Cunha, F. L., \& Melani, R. F. H. (2008). Avaliação dos mestrandos em ortodontia: Utilização dos documentos que compõe o prontuário odontológico. Rev Odont Acad Tiradentes Odont, 10, 537-567.

Código de Ética Odontológica (2003). Resolução CFO - 42/2003. http://www.portaldabioetica.com.br/legislacao/45.

Ferreira Cruz, S., Penha Mathias, A., De Souza Viana, B. A., De Souza Silva, N., Ducatti Alves, M. \& Fernando Lolli, L. (2017). Esclarecendo a responsabilidade civil do cirurgião-dentista. Acta Jus: Periódico da Área de Direito, 11(1).

Hupp, J. R., Ellis, E. T., \& MR, C. (2015). Oral e maxilofacial contemporânea. Elsevier.

Kato, M. T., Goya, S., Peres, S. H. De C. S., Peres, A. S., \& de Magalhães Bastos, J. R. (2018). Responsabilidade civil do cirurgião-dentista. Revista de Odontologia da Universidade Cidade de São Paulo, 20(1), 66-75.

Lima Amorim, H. P., Marmol, S. L. P., Cerqueria, S. N. N., da Silva, M. L. C. A., \& da Silva, U. A. (2016). A importância do preenchimento adequado dos prontuários para evitar processos em Odontologia. Arquivos em Odontologia, 52(1).

Machado, M. S., Nagano, H. C. M., da Silva, J. Y. B., \& Bosco, V. L. (2017). Participação dos pais na tomada de decisões no atendimento odontológico de seus filhos. Revista de Odontologia da Universidade Cidade de São Paulo, 21(1), 38-47.

Ministério da Saúde, Conselho Nacional de Saúde (2013). Comissão Nacional de Ética em pesquisa. Res. CNS nº 466/2012. Brasília, 2013. Disponível em: http://conselho.saude.gov.br/resolucoes/2012/reso466.pdf.

Mota, J. M. S., Araújo, M. E. De, Carrer, F. C. De A., Lopez-Capp, T. T., Biazevic, M. G. H., \& Michel-Crosato, E. (2018). Personal and professional profile, education, and perception of management and entrepreneurship of Dentistry students. Rev. ABENO, 85-94.

Paranhos, L. R., Caldas, J. C. F., Iwashita, A. R., Scanavini, M. A., \& de Cassia Paschini, R. (2009). A importância do prontuário odontológico nas perícias de identificação humana. Revista da Faculdade de Odontologia-UPF, 14(1).

Saraiva, A. S. (2012). A importância do prontuário odontológico-com ênfase nos documentos digitais. Revista Brasileira de Odontologia, $68(2), 157$.

Souza, J. J. De, Fernandes, L. A., \& others. (2021). Análise dos aspectos éticos e jurídicos do prontuário odontológico. Revista Direito UTP, 2(1), 63-75.

Vanrell, J. P. (2012). Odontologia legal \& Antropologia Forense. Ed. Guanabara Koogan. 